\title{
Signification de la satiété à court terme chez le mouton : Influence de la qualité du fourrage et des stimuli associés à la prise de nourriture
}

\author{
F. GATEL \\ avec la collaboration technique de L. L'HoteliER \\ I.N.R.A., C.R.Z.V. de Theix, Laboratoire des Aliments \\ Centre de Recherches de Clermont-Ferrand, F 63122 Ceyrat
}

\begin{abstract}
Résumé
Quatre béliers castrés recevaient à volonté deux fois par jour du foin de ray-grass de qualité médiocre ou du foin de luzerne de bonne qualité. Après avoir ingéré un premier repas, ils recevaient de nouveau, en quantité non limitée, soit le même fourrage, soit l'autre. La fin du second repas était définie comme pour le premier par un temps d'arrêt de l'ingestion de cinq minutes.

Un arrêt de l'ingestion de cinq minutes n'exclut pas la possibilité d'une reprise immédiate : l'ingestion est généralement déclenchée lorsqu'on remet du fourrage dans l'auge de l'animal.

Quel que soit le repas, la durée d'ingestion est plus longue et la vitesse d'ingestion plus élevée avec la luzerne qu'avec le ray-grass, si bien que les quantités ingérées sont également plus élevées. Ces différences se trouvent amplifiées lorsque le fourrage ingéré lors du second repas est différent du fourrage ingéré lors du premier repas.
\end{abstract}

Mots clés : Ruminants, comportement alimentaire, repas, satiété à court terme, qualité du fourrage.

\section{Introduction}

L'ingestion des aliments par les animaux a pour objet de compenser les pertes d'entretien et de production; la satiété intervient cependant bien avant la fin de l'adsorption des nutriments, avant donc que les déficits ne soient comblés, et correspond à un mécanisme d'anticipation, l'animal étant capable d'établir une relation entre l'effet sensoriel des aliments aux niveaux buccal et gastrique et leurs effets métaboliques (Le Bars, Gallouin, 1972).

Par ailleurs, chez des animaux recevant des rations à faible densité énergétique, l'ingestion peut être limitée par la capacité physique des réservoirs digestifs. Cela est particulièrement vrai pour les ruminants recevant des fourrages grossiers, pour lesquels on considère généralement que l'arrêt de l'ingestion se produit lorsque les pré-estomacs 
(rumen et réseau principalement) atteignent un certain niveau de réplétion (cf. revues de Balch \& Campling, 1962 ; Campling, 1970 ; Baile \& Forbes, 1974 ; Gallouin \& Focant, 1980). Avec ce type de fourrage, l'ingestion est limitée par la capacité du rumen-réseau et par le volume occupé par les digesta à la fin du repas. Cependant, on observe souvent qu'un ruminant qui vient de s'arrêter de manger, et est donc apparemment rassasié, se remet à manger lorsqu'on lui présente à nouveau du fourrage. De même, l'application à des rats rassasiés de stimuli normalement associés à la prise de nourriture peut déclencher à nouveau celle-ci (WEINGARTEN, 1983).

Enfin, les quantités ingérées peuvent dépendre de l'appétibilité du fourrage distribué, indépendamment de ses qualités digestives et nutritionnelles (ARNOLD, 1966; Greenhalgh \& Reid, 1967 et 1971 ; Van Niererk, Greenhalgh \& Reid, 1973).

Ainsi, même si certains facteurs comme la réplétion du rumen-réseau gardent un rôle prépondérant sur l'établissement de la satiété à court terme chez les ruminants, d'autres phénomènes peuvent intervenir pour moduler les quantités ingérées ou favoriser le déclenchement de l'ingestion. Le but de cette expérience était d'étudier plus précisément, chez des moutons adultes, les interactions pouvant exister entre l'arrêt de l'ingestion, le type de fourrage, et certains stimuli normalement associés au déclenchement de la prise de nourriture.

\section{Matériel et méthodes}

Quatre béliers castrés, de race Texel, pesant en moyenne $66,5 \mathrm{~kg}$ ont été alimentés individuellement à volonté (deux distributions d'aliment par jour, à $8 \mathrm{~h}$ et $16 \mathrm{~h}$ ) selon le protocole suivant : du fourrage en excès était placé dans l'auge et l'animal mangeait un premier repas ( $\mathrm{R} 1$ ) ; ce premier repas était considéré comme terminé lorsque l'animal s'était arrêté de manger pendant cinq minutes au moins ; les refus étaient alors enlevés, et du fourrage en excès placé à nouveau dans l'auge. Le second repas (R2) était défini selon le même critère que le premier. A la fin du second repas, les refus étaient également enlevés et on remettait dans l'auge les refus du premier repas, jusqu’à la distribution suivante. L'eau était disponible en permanence à volonté.

Les fourrages utilisés étaient deux foins de nature et de qualité très différentes, un bon foin de luzerne ( $L$ ) et un mauvais foin de ray-grass italien ( $R$ ) ; les digestibilités de la matière organique étaient respectivement 62 p. 100 et 54,5 p. 100 , et les compositions chimiques étaient en p. 100 de la matière sèche respectivement les suivantes : matière organique : 89,6 et 94,3 ; matières azotées : 19,7 et 5,8 ; cellulose brute : 29,1 et 35,4 .

Les foins étaient utilisés séparément, le même foin étant ingéré lors des repas $R 1$ et $R 2$, ou en association, les repas $R 1$ et $R 2$ correspondant aux deux foins différents. Chaque combinaison foin $\times$ numéro de repas a été appliquée quatre fois à chacun des quatre animaux. Pour éviter que les animaux ne s'habituent aux modalités de distribution du fourrage, les mesures n'avaient lieu qu'un jour sur deux au maximum, et en alternant le fourrage distribué pour le repas R2. Les jours sans mesure, les animaux ne recevaient que deux distributions à $8 \mathrm{~h}$ et $16 \mathrm{~h}$ du même fourrage. 
Nous avons également enregistré les mouvements de mâchoire des animaux, selon la méthode de Ruckebusch (1963), de façon à connaître la durée des repas et la vitesse d'ingestion (calculée en rapportant la quantité ingérée à la durée d'ingestion). Cet enregistrement nous permettait en outre d’apprécier la fin de chaque repas.

\section{Résultats (fig. 1)}

Lorsque les animaux ne reçoivent qu'un seul des deux fourrages (traitements LL ou RR), la durée journalière d'ingestion est de $389 \pm 50 \mathrm{mn}$ pour la luzerne et de $302 \pm 46 \mathrm{mn}$ pour le ray-grass. Les quantités consommées par jour sont de $91 \pm 11 \mathrm{~g}$ $\mathrm{MS} / \mathrm{kg} \mathrm{P}^{0.75}$ pour la luzerne et de $43 \pm 6 \mathrm{~g} \mathrm{MS} / \mathrm{kg} \mathrm{P}^{0.75}$ pour le ray-grass, les vitesses

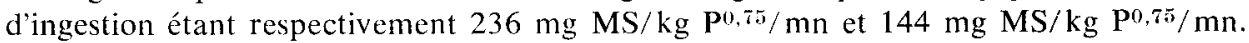
Les quantités consommées par jour sont significativement plus élevées $(P<0,01)$ que les quantités normalement ingérées lorsqüil n'y a que deux distributions quotidiennes de fourrage.

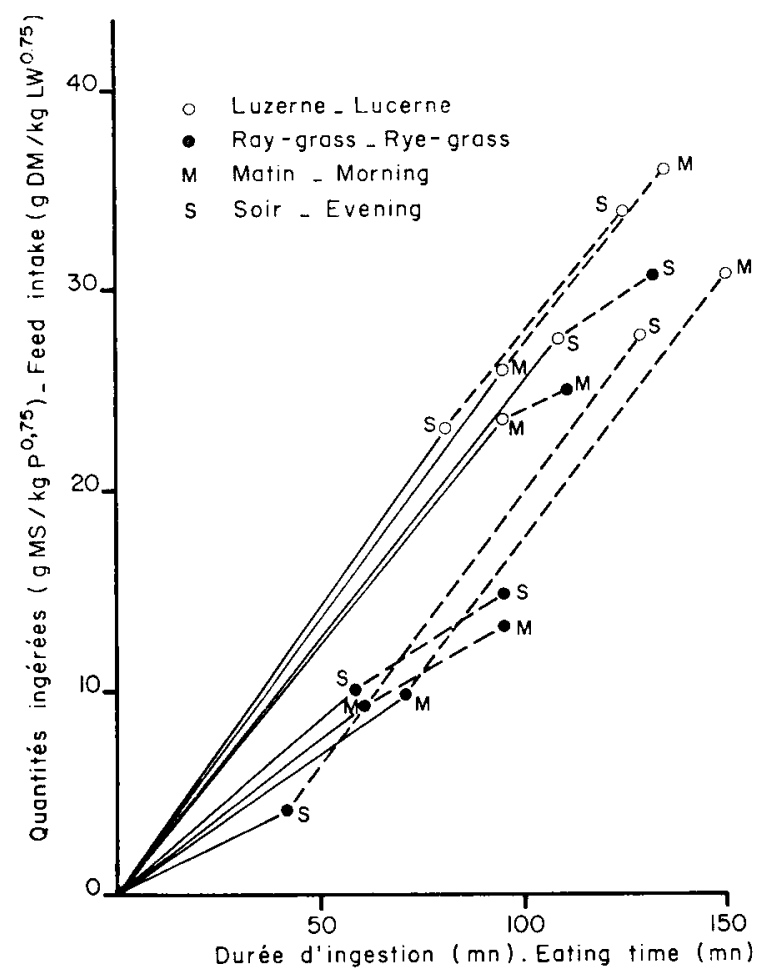

FIG. 1

Quantités ingérées et durée d'ingestion lors du premier (-) et du second (- -) repas.

La pente de la droite représente la vitesse d'ingestion moyenne au cours du repas.

Feed intake and eating time (first — and second ... meal). Slope indicates mean eating rate during the meal. 
Quel que soit le fourrage, les quantités ingérées lors du repas $\mathrm{R} 2$ représentent de 38 à 46 p. 100 du repas $R 1$. Le rapport $R 2 / R 1$ est donc du même ordre de grandeur pour les deux fourrages, et est un peu plus élevé le soir que le matin.

\section{A. Repas $R I$}

La durée d'ingestion correspondant à ce premier repas est en moyenne de $77 \pm$ $31 \mathrm{mn}$; ce repas est significativement plus long $(\mathrm{P}<0,01)$ avec la luzerne $(95 \pm 27 \mathrm{mn})$ qu'avec le ray-grass $(59 \pm 25 \mathrm{mn})$. La vitesse d'ingestion est également significativement plus élevée $(\mathrm{P}<0,01)$ avec de la luzerne $\left(273 \pm 56 \mathrm{mg} \mathrm{MS} / \mathrm{kg} \mathrm{P}^{0,75} / \mathrm{mn}\right)$ qu'avec du ray-grass $\left(147 \pm 49 \mathrm{mg} \mathrm{MS} / \mathrm{kg} \mathrm{P}^{0,75} / \mathrm{mn}\right)$. Aussi les quantités ingérées lors de ce premier repas, qui sont en moyenne de $17 \pm 10 \mathrm{~g} \mathrm{MS} / \mathrm{kg} \mathrm{P}^{0,75}$, varient-elles surtout $(\mathrm{P}<0,01)$ avec le type de foin (66 p. 100 de la variabilité expliquée par ce facteur) : $25 \pm 7 \mathrm{~g} \mathrm{MS} / \mathrm{kg} \mathrm{P}^{0,75}$ pour la luzerne, $9 \pm 4 \mathrm{~g} \mathrm{MS} / \mathrm{kg} \mathrm{P}^{0,75}$ pour le ray-grass.

Par ailleurs, qu'il s'agisse de la durée d'ingestion, de la vitesse d'ingestion ou de la quantité ingérée, on ne note pas de différences significatives entre les repas $\mathbf{R} 1$ du matin et du soir, ni selon le fourrage distribué après $\mathrm{R} 1$.

\section{B. Repas $R 2$}

Les quantités ingérées lors de ce second repas sont, en moyenne, de $10 \pm 8 \mathrm{~g}$ $\mathrm{MS} / \mathrm{kg} \mathbf{P}^{0,75}$.

Comme pour le repas $\mathrm{R} 1$, la nature du fourrage constitue la principale source de variation : quel que soit le fourrage ingéré lors du repas $R 1$, le repas $R 2$ dure plus longtemps $(63 \pm 32 \mathrm{mn}$ contre $26 \pm 18 \mathrm{mn})$, la vitesse d'ingestion est plus forte $\left(278 \pm 85 \mathrm{mg}\right.$ contre $\left.110 \pm 58 \mathrm{mg} \mathrm{MS} / \mathrm{kg} \mathrm{P}^{0,75} / \mathrm{mn}\right)$ et les quantités ingérées sont plus élevées $\left(16 \pm 7 \mathrm{~g} \mathrm{MS} / \mathrm{kg} \mathrm{P}^{0,75}\right.$ contre $\left.3 \pm 2 \mathrm{~g} \mathrm{MS} / \mathrm{kg} \mathbf{P}^{0,75}\right)$ avec de la luzerne qu'avec du ray-grass (différences significatives, $\mathbf{P}<0,01$ ).

D'autre part, on constate un effet du fourrage ingéré lors du repas R1 sur le repas R2 : celui-ci dure plus longtemps, l'ingestion est plus rapide et les quantités ingérées sont plus importantes lorsque les animaux ont précédemment ingéré, lors du repas $\mathrm{R} 1$, du ray-grass que de la luzerne (différences significatives, $\mathbf{P}<0,01$ ).

Enfin, les quantités ingérées lors du repas $R 2$ sont un peu plus élevées $(P<0,05)$ le soir que le matin. En revanche, les durée et vitesse d'ingestion ne varient pas de façon significative entre le matin et le soir.

\section{Discussion et conclusion}

L'existence du repas $R 2$ (qui a effectivement eu lieu dans 120 des 128 observations) montre qu'un animal qui s'est arrêté de manger depuis $5 \mathrm{mn}$ est capable de se remettre à manger lorsqu'on remet du fourrage dans son auge. Cependant, l'appré- 
ciation de la fin du repas soulève, chez les ruminants, un problème qui n'est pas vraiment résolu : la plupart des auteurs considèrent le repas terminé avec l'apparition d'un temps de repos supérieur à un certain seuil ; la valeur de ce seuil, fixée arbitrairement, varie de $5 \mathrm{mn}$ (Skouri, 1966) à $20 \mathrm{mn}$ (Baile, 1975; Forbes, 1980). DURANTON et al. (1983) proposent plutôt l'apparition de la première période de rumination comme critère attestant la fin du repas. Suzuki, Shinde, Hidari (1973), étudiant les variations de la vitesse d'ingestion avec une méthode de double repas similaire à la nôtre distribuaient à nouveau le fourrage lorsque les animaux avaient cessé de manger depuis $5 \mathrm{mn}$. Toutefois ce temps d'arrêt de l'ingestion de $5 \mathrm{mn}$ n'est probablement pas assez long pour signifier que les animaux avaient atteint un état de "satiété », d'autant que nous n'avions pas d'animaux à qui nous ne renouvellions pas le fourrage. Il indique plus vraisemblablement une diminution de la propension à manger. Mais nous constatons que les stimuli habituels de début de l'ingestion chez des animaux alimentés à l'auge, enlèvement des refus et présentation d'une nouvelle quantité d'aliments (Dulphy, Remond, Theriez, 1980) ont généralement été capables d'inhiber cette diminution. En associant chez des rats la distribution d'aliment à un signal sonore, WEINGARTEN (1983) avait déjà constaté que ce même stimulus déclenche chez les rats rassasiés une reprise très significative de l'ingestion : (les quantités ingérées lors de ce second repas représentent 20 p. 100 de lingéré quotidien) ; cependant, le rat diminue spontanément les repas suivants, de sorte que sa consommation quotidienne n'est pas modifiée. Dans notre essai, lorsque les repas $\mathrm{R} 1$ et $\mathrm{R} 2$ correspondent au même fourrage (traitement $\mathbf{L L}$ ou $R \mathrm{R}$ ), le repas $\mathrm{R} 2$ ne représente que 11 p. 100 environ de l'ingéré quotidien. Les ruminants, une fois proches de la satiété, seraient peut-être, du fait de la limitation exercée par l'encombrement de leurs réservoirs gastriques, moins sensibles à ce type de conditionnement que les monogastriques; d'autre part, contrairement à ce qui se passe chez les rats, les quantités ingérées par jour restent significativement $(\mathbf{P}<0,01)$ supérieures aux quantités normalement ingérées lorsqu'il n’y a que deux distributions quotidiennes de fourrage. Les mesures n'avaient d'ailleurs lieu qu'un jour sur deux au maximum, de façon à permettre aux animaux de revenir à un état plus normal.

Quel que soit le repas ( $\mathrm{R} 1$ ou $\mathrm{R} 2$ ), on constate que la luzerne est toujours consommée en plus grande quantité que le ray-grass. Cette différence ne peut être due qu'aux caractéristiques propres des fourrages et elle est amplifiée, lors du repas R2, par l'effet «succession des fourrages»: ainsi, les animaux consomment, lors du repas R2, plus de luzerne lorsqu'elle est distribuée après du ray-grass qu'après de la luzerne, et moins de ray-grass lorsqu'il est distribué après de la luzerne qu'après du ray-grass; un des quatre animaux a même presque toujours ( 7 fois sur 8 ) refusé de manger du ray-grass lorsqu’il était distribué après de la luzerne. Ceci montre que la propension à manger d'un animal qui vient de s'arrêter de manger pendant $5 \mathrm{mn}$ dépend non seulement du fourrage qu'on lui présente, mais aussi de celui qu'il vient d'ingérer, et de la façon dont ces deux fourrages se situent qualitativement l'un par rapport à l'autre.

Quel que soit le repas, la différence des quantités ingérées cntre la luzerne et le ray-grass est liée à la fois à une durée d’ingestion plus longue et à une vitesse d"ingestion plus élevée dans le cas de la luzerne. Dulphy \& Demarquil.ly (1974), DulPhy \& Bechet (1976), avec des fourrages verts avaient déjà montré l'influence des caractéristiques du fourrage (famille botanique, espèce, stade de végétation) sur la durée et surtout la vitesse d'ingestion, ainsi que la liaison entre ces deux paramètres 
et les quantités ingérées ; ces différences ne semblaient pas liées à des différences dans les teneurs en azote ou en cellulose brute. Avec des fourrages secs de légumineuses, Peterson, Baumgardt \& Long (1974) ont observé que la vitesse d'ingestion était proportionnelle $(\mathrm{P}<0,01)$ à la densité du fourrage (exprimée en $\mathrm{g} \mathrm{MS} / \mathrm{ml}$ ) et émis l'hypothèse qu'une fois le repas commencé, la vitesse d'ingestion était limitée par le volume du fourrage ingéré (la vitesse d'ingestion exprimée en $\mathrm{ml} / \mathrm{mn}$, ne présentait plus de différence entre les fourrages). Ceci pourrait expliquer, en partie du moins, les différences que nous constatons : sans avoir mesuré rigoureusement la densité de nos fourrages, nous avons constaté que la luzerne était plus dense que le ray-grass. Cette différence de densité pourrait également contribuer à expliquer la différence de quantités ingérées entre la luzerne et le ray-grass, comme l'ont montré SEOANE, Cote, VisSER (1982).

Pour l'ensemble des quatre traitements : LR, LL, RR et RL, nous n'avons pas observé d'influence significative du numéro du repas (R1 ou R2) sur la vitesse d'ingestion. Pourtant, on constate habituellement que la vitesse d'ingestion diminue au cours du repas, au fur et à mesure que l'animal se rassasie (Duckworth \& SHIRlaw, 1958 ; Forbes \& Wright, 1968 ; Suzuki, Fujita, Shinde, 1969 ; Forbes, Wright, Bannister, 1972 ; Suzuki, Shinde, Hidari, 1973 ; Owen et al., 1976 ; Deswysen \& Ehrlein, 1981). Cette diminution résulte à la fois d'une augmentation du nombre et de la durée des périodes de pause (Duckworth \& Shirlaw, 1958 ; Cirio Maisonnave, Boivin, Bost, 1980 et 1981 ; Deswysen \& Ehrlein, 1981) et d'une diminution de l'intensité de la mastication - paramètre qui tient compte de la fréquence des mouvements masticatoires et de leur amplitude - (Cirio Marsonnave, Boivin, Bost, 1980 et 1981), et traduit une diminution de la motivation des animaux. Les stimuli habituels du début de l'ingestion semblent cependant avoir eu un effet prépondérant sur l'état de faim/satiété, puisque la vitesse d'ingestion moyenne est la même lors du premier et du second repas. Toutefois, nous ne disposons que d'une vitesse d'ingestion moyenne par repas, et les enregistrements de mouvements de mâchoires réalisés ne nous permettent pas d'apprécier l'évolution de l'intensité de la mastication lors des repas, si bien que nous ne pouvons pas comparer la façon dont se déroulent les repas $\mathrm{R} 1$ et $\mathrm{R} 2$. D'autre part, la vitesse d'ingestion moyenne tient compte des périodes de pause pendant lesquelles l'animal ne mastique pas; le choix d'un critère de fin de repas peut-être trop court peut aussi avoir introduit une erreur dans l'estimation de la vitesse d’ingestion. Quoiqu'il en soit, avec des vaches taries, recevant du foin ou du fourrage vert, Suzuki, Shinde, Hidari (1973) avaient déjà constaté en distribuant chaque repas en deux moitiés (la seconde moitié étant distribuée lorsque l'animal s'était arrêté de manger pendant $5 \mathrm{mn}$ ) un maximum de vitesse d'ingestion au début de chaque «demi-repas». Cependant, dans notre étude, si l'on considère le ray-grass seul, la vitesse d'ingestion est plus élevée $(\mathrm{P}<0,01)$ lors du repas $\mathrm{R} 1$ que lors du repas R2 : $149 \mathrm{mg} \mathrm{MS} / \mathrm{kg} \mathrm{P}^{0,75} / \mathrm{mn}$ contre $111 \mathrm{mg} \mathrm{MS} / \mathrm{kg} \mathrm{P}^{0,75} / \mathrm{mn}$; avec du fourrage peu appétible, l'état de faim ou de satiété garderait donc, peut-être, un effet prépondérant sur la vitesse d’ingestion.

Enfin, on observe peu de différences de comportement alimentaire entre les distributions du matin et du soir, celles-ci n'étant significatives que pour les quantités ingérées lors du second repas; en ce qui concerne le repas $\mathrm{R} 1$, seuls les animaux soumis au traitement RL consomment moins de ray-grass le soir, par suite sans doute de la consommation importante de luzerne lors du repas R2 le matin. Les vitesses d'ingestion par contre sont toujours les mêmes. Le protocole adopté lors de chaque 
distribution contribue peut-être à supprimer les différences généralement observées lorsque les animaux sont plus libres de leur comportement (la vitesse d'ingestion est généralement plus élevée le soir, et les quantités ingérées le sont parfois aussi).

L'établissement de la satiété à court terme n'a donc qu'une signification relative : il dépend de la qualité du fourrage mais aussi d'autres facteurs comme la présence de stimuli normalement associés à la prise de nourriture.

\author{
Summary \\ Signification of initial satiety in sheep : \\ effects of hay quality and cues associated with feeding
}

Four wethers were fed twice daily with either poor quality rye-grass hay or good quality lucerne hay, ad libitum. Right after the first meal immediately after feeding, they were again offered unrestricted quantities of the same or the other hay. A meal was considered to be over once the sheep stopped all ingestive activities for five minutes.

A five minutes interruption in intake did not mean that the animal could not resume eating; however, this meal criterion was probably not long enough to indicate that the sheep was actually satiated.

For either the first or the second meal, feed intake was higher $(P<0.01)$ with lucerne than with rye-grass (fig. 1); this was due to a longer eating time and a higher eating rate (fig. 1). These differences between the two hays should depend on hay properties, such as palatability or bulk density. In addition, they were amplified during the second meal when the roughage eaten was different from the roughage eaten at the first meal. These results suggest that the inclination to eat in an animal that has stopped cating for five minutes depends not only on the quality of the roughage given, but also on the quality of the roughage it has previously been fed.

Key words : Ruminants, feeding behaviour, meal, initial satiety, roughage quality.

Reçu en mai 1893.

Accepté en octobre 1983.

\title{
Références bibliographiques
}

ARNold G.W., 1966. The special senses in grazing animals. II. Smell, taste and touch and dietary habits in sheep. Aust. J. Agric. Res., 17, 531-542.

Baile C.A., Forbes M., 1974. Control of feed intake and regulation of encrgy balance in ruminants. Physiol. Rev., 54, 160-214.

Baile C.A., 1975. Control of feed intake in ruminants. in Mc Donald I.W., Warner A.C.I., Ed. Digestion and Metabolism in ruminants. Proc. Fourth Intern. Symposium, Sydney (Australia), 1974, University of New England, Pub. Unit, 333-350.

Balch C.C., Campling R.C., 1962. Regulation of voluntary food intake in ruminant. Nutr. Abstr. Rev., 32, 669-686.

Campling R.C., 1970. Physical regulation of voluntary intake. in Phillipson A.T., Ed. Physiology of digestion and metabolism in ruminants. Proc. Third Int. Symposium, Cambridge, England, 1969. Oriel Press., 226-234. 
Cirio Maisonnave A., Boivin R., Bost J., 1980. Comportement alimentaire et mérycique chez le mouton : effets de la nalorphine par voie intracérébro-ventriculaire. Ann. Rech. Vét., 11, 57-67.

Cirio Maisonnave A., Borvin R., Bost J., 1981. Stimulation prandiale de la motricité réticulaire chez le mouton : phase cépalique et réflexe oral. Ann. Rech. Vét., 12, 291-302.

Deswysen A.G., Ehrlein N.J., 1981. Silage intake, rumination and pseudo-rumination activity in sheep studied by radiography and jaw movement recordings. Br. J. Nutr., 46, 327-335.

Duckworth J.E., Shirlaw D.W., 1958. A study of factors affecting feed intake and the eating behaviour of cattle. Anim. Behav., 6, 147-154.

Dulphy J.P., Bechet G., 1976. Influence du stade de végétation et de l'espèce végétale sur le comportement alimentaire et mérycique de moutons recevant des fourrages verts hachés. Ann. Zootech., 25, 505-519.

Dulphy J.P., Demarquilly C., 1974. Etude du comportement alimentaire et mérycique de moutons recevant des fourrages verts hachés. Ann. Zootech., 23, 193-212.

Dulphy J.P., REMOND B., Theriez M., 1980. Ingestive behaviour and related activities in ruminants. in Ruckebusch Y., Thivend P., Ed. Digestive Physiology and Metabolism in Ruminants. Proc. Fifth Intern. Symposium. Clermont-Ferrand (France), 1979. MTP Press Limited, 103-122.

Duranton A., Bueno L., Ruckebusch Y., 1983. Recherches sur la satiété à court terme chez les ruminants. Rev. Méd. Vét., 134, 11-18.

Forbes J.M.., Wright J.A., 1968. Rate of eating in sheep. Anim. Prod., 10, 240 (Abstr.).

Forbes J.M., Wright J.A., Bannister A., 1972. A note on rate of eating in sheep. Anim. Prod., 15, 211-214.

Forbes J.M., 1980. A model of the short term control of feeding in the Ruminant : effects of changing animal or feed characteristics. Apppetite, 1, 21-41.

Gallouin F., Focant M., 1980. Bases physiologiques du comportement alimentaire chez les ruminants. Reprod. Nutr. Dévelop., 20, 1563-1614.

Greenhalgh J.F.D., Reid G.W., 1967. Separating the effects of digestibility and palatability on food intake in ruminants animals. Nature, 214, 744-745.

Greenhalgh J.F.D., Reid G.W., 1971. Relative palatability to sheep of straw, hay and dried grass. Br. J. Nutr., 26, 107-116.

Le Bars H., Gallouin F., 1972. Bases physiologiques du comportement alimentaire. in Association Internationale Vétérinaire de Production Animale, Ed., $2^{\circ}$ Congrès Mond. Alimentation animale, Madrid (Espagne), $\mathrm{n}^{\circ} 4,671-702$.

Owen G.L., Martz F.A., Campbell J.R., Matches A.G., Hilderbrand E.S., 1976. Relation of eating and associated behavioural patterns in cattle in confinement to forage species and ambient temperature. J. Anim. Sci., 42, 1534-1540.

Peterson A.D., Baumgart B.R., Long T.A., 1974. Relationship between intake of some forage and feeding behaviour of sheep. J. Anim. Sci., 38, 172-177.

Ruckebusch Y., 1963. Recherches sur la régulation centrale du comportement alimentaire chez les ruminants. Thèse Doct. Sci. Nat., Lyon (France), 215 p.

SEOANE J.R., COTE M., VisSer S.A., 1982. The relationship between voluntary intake and the physical properties of forages. Can. J. Anim. Sci., 62, 473-480.

Skouri M., 1966. Valeur nutritive de la ration et comportement alimentaire du ruminant. Thèse Doct.-Ingénieur, Paris (France), 175 p.

Suzuki S., Fujita H., Shinde Y., 1969. Change in the rate of eating during a meal and the effect of the interval between meals in the rate at which cows eat roughages. Anim. Prod., 11, 29-41.

Suzuki S., Shinde Y., Hidari H., 1973. Effect of divided feeding of roughage on the rate of eating during a meal in dairy cows. Jap. J. Zootech. Sci., 44, 181-187.

Van Niererk A.I., Greenhalgh J.F.D., Reid G.W., 1973. Importance of palatability in determining the feed intake of sheep offered chopped and pelleted hay. Br. J. Nutr., 30, 95-105.

Weingarten H.P., 1983. Conditioned cues elicit feeding in sated rats : a role for learning in meal initiation. Science, 220, 431-433. 